ISSN 1808-3765

\title{
AVALIAÇÃO DA UNIFORMIDADE DE APLICAÇÃO DE ÁGUA EM GOTEJADORES EM FUNÇÃO DO TEOR DE FERRO
}

\author{
Rigléia Lima Brauer ${ }^{1}$; Raimundo Leite $\mathrm{Cruz}^{2}$; Roberto Lyra Villas Bôas ${ }^{3}$; Talita \\ Aparecida Pletsch ${ }^{1}$ \\ ${ }^{1}$ Doutoranda, Departamento de Engenharia Rural, Faculdade de Ciências Agronômicas, Universidade \\ Estadual Paulista, Botucatu, SP, rigleia@fca.unesp.br \\ ${ }^{2}$ Professor Doutor, Departamento de Engenharia Rural, Faculdade de Ciências Agronômicas, Universidade \\ Estadual Paulista, Botucatu, SP \\ 3 Professor Doutor, Departamento de Recursos Naturais Ciência dos Solos, Faculdade de Ciências \\ Agronômicas, Universidade Estadual Paulista, Botucatu/SP
}

\section{RESUMO}

Este trabalho teve por objetivo estudar a variação de vazão em tubogotejadores NaanPC laranja e NaanPC azul em função de precipitados químicos, ao longo do tempo de funcionamento, quando submetidos a uma água rica em ferro, avaliando-se especificamente o coeficiente de uniformidade de Christiansen (CUC) e o coeficiente de uniformidade de distribuição (CUD). O experimento foi desenvolvido em uma bancada de ensaios para tubogotejadores no Laboratório de Ensaio de Equipamentos para Irrigação do Departamento de Engenharia Rural da Faculdade de Ciências Agronômicas - UNESP, localizado no município de Botucatu - SP. O experimento foi montado em esquema fatorial $2 \times 2 \times 7$, sendo duas doses de $\mathrm{Fe}$, dois modelos de gotejadores e sete tempos de funcionamento, com três repetições, totalizando 84 parcelas experimentais. Efetuou-se a análise de variância pelo teste F. As médias das vazões dos gotejadores foram comparadas pelo teste de T de Student a 5\% de probabilidade. Os resultados obtidos não apontaram diferença significativa nas diferentes doses de ferro. Entretanto, os resultados apontaram diferença significativa na interação gotejador x tempo e coeficiente de uniformidade de Christiansen (CUC).

UNITERMOS: coeficiente de uniformidade de Christiansen(CUC), coeficiente de uniformidade de distribuição (CUD), entupimento de emissores.

\section{BRAUER, R.L; CRUZ, R. L; VILLAS BÔAS, R. L.; PLETSCH, T. A. EVALUATION OFWATER APPLICATION UNIFORMITY IN DRIPPERSIN FUNCTION OF IRON CONTENT}

\section{ABSTRACT}

This work aimed to study the occurrence of chemical precipitates effects in two types of drip hose (NaanPC orange and blue) due to chemical precipitation along the operating time, when subjected to water rich in iron, specifically evaluating the Christiansen uniformity coefficient (CUC) and distribution uniformity (DU). The experiment was conducted in a test bench for drip hose at Laboratory Test Equipment for Irrigation - Department of Agricultural Engineering, Sao Paulo State University. The experiment was a 2 × 2 × 7 factorial design, with two Fe doses, two drip brands, and seven operational times with three replicates, 
totalizing 84 experimental plots. The variance analysis was performed by $\mathrm{F}$ test. Average emitter discharges were compared by Student $t$ test at $5 \%$ probability. The results showed no significant difference in iron doses. However, the results indicated significant differences in drip versus operational time and the Christiansen uniformity coefficient (CUC).

KEYWORDS: Uniformity coefficient of Christiansen(CUC), distribution uniformity (DU), emitter clogging.

\section{INTRODUÇÃO}

A irrigação localizada representa um avanço na tecnologia de irrigação. Pode ser definida como uma aplicação precisa de água na forma de gotas, através de emissores, localizados em pontos selecionados ao longo das linhas condutoras de água, e se bem manejado resulta na economia de água e energia. A qualidade da água é um fator essencial, pois problemas com entupimento diminuem a eficiência do sistema. Desta forma análises das fontes de água são necessárias, exigindo-se água de boa qualidade, com baixo nível de sólidos suspensos e solúveis, como o ferro, que pode precipitar e causar problemas no sistema, sendo aconselhável sua análise antes da instalação do sistema de gotejamento.

A qualidade da água de irrigação está diretamente relacionada com a obstrução de emissores (Povoa; Hills, 1994), a qual recebe diversas classificações, onde uma destas pode ser observada na Tabela 1 desenvolvida por Bucks et al. (1979), que tem por objetivo proporcionar uma orientação de caráter quantitativo, indicando critérios para avaliação do risco de entupimento de emissores.

Tabela 1. Propostas de classificação do potencial de risco das águas no entupimento de sistemas de irrigação localizada

\begin{tabular}{ccccc}
\hline Causa obstrutora potencial & Unidade & \multicolumn{3}{c}{ Grau de restrição ao uso } \\
\cline { 3 - 5 } & & Nenhum & Leve a moderado & Severo \\
\hline Sólidos suspensos & $\mathrm{mg} \mathrm{L}^{-1}$ & $<50$ & $50-100$ & $>100$ \\
$\mathrm{pH}$ & $\mathrm{mg} \mathrm{L}^{-1}$ & $<7.0$ & $7.0-8.0$ & $>8.0$ \\
Sólidos dissolvidos & $\mathrm{mg} \mathrm{L}^{-1}$ & $<500$ & $500-2.000$ & $>2.000$ \\
Manganês & $\mathrm{mg} \mathrm{L}^{-1}$ & $<0.1$ & $0.1-1.5$ & $>1.5$ \\
Ferro & $\mathrm{mg} \mathrm{L}^{-1}$ & $<0.1$ & $0.1-1.5$ & $>1.5$ \\
Sulfeto de hidrogênio & $\mathrm{mg} \mathrm{L}^{-1}$ & $<0.5$ & $0.5-2.0$ & $>2.0$ \\
População bacteriana & $\mathrm{N}^{\mathrm{o}} \mathrm{Máximo}^{*}$ & $<10.000$ & $10.000-50.000$ & $>50.000$ \\
\hline
\end{tabular}

*Unidades Formadores de Colônia

Fonte: Bucks, Nakayama e Gilbert (1979).

Para Reis et al (2002), a avaliação do desempenho de um sistema de irrigação é uma etapa fundamental antes que qualquer estratégia de manejo de irrigação seja implementada. MANTOVANI; RAMOS (1994) afirmam que a uniformidade da irrigação tem como objetivo básico melhorar a produtividade e/ou a rentabilidade da propriedade. 
Segundo Bernardo (1995), é importante determinar a uniformidade de distribuição de água em qualquer método de irrigação após a instalação do sistema e a cada dois anos de funcionamento.

Sabe-se que a questão do entupimento de emissores na irrigação por gotejamento é uma realidade no campo. Tendo em vista esta questão, neste trabalho objetivou-se a estudar o efeito da ocorrência de precipitados químicos em dois tipos de tubo gotejadores (NaanPC laranja e NaanPC azul), em função do tempo de funcionamento, quando submetidos a uma água rica em ferro, avaliando-se especificamente o coeficiente de uniformidade de Christiansen (CUC) e coeficiente de uniformidade de distribuição (CUD).

\section{MATERIAIS E MÉTODOS}

O experimento foi conduzido no Laboratório de Ensaio de Equipamentos para Irrigação do Departamento de Engenharia Rural da Faculdade de Ciências Agronômicas UNESP, localizada no município de Botucatu - SP, utilizando-se uma bancada de ensaios para tubogotejadores com $6,0 \mathrm{~m}$ de comprimento por 1,86m de largura (Figura 1).

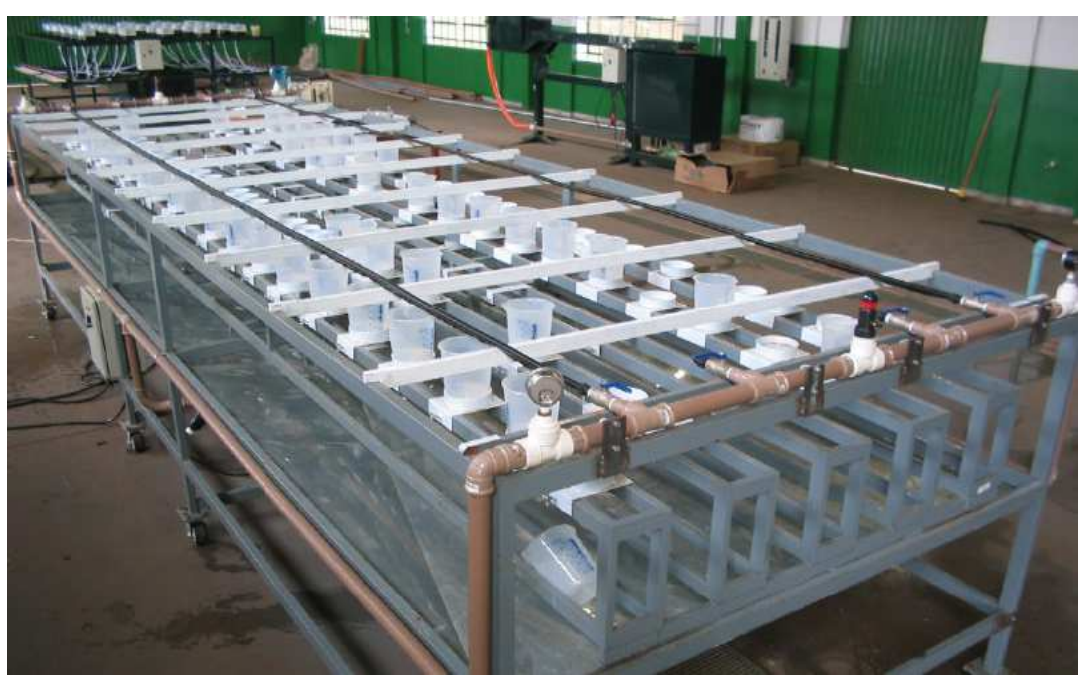

Figura 1. Detalhe do experimento conduzido na bancada de ensaios.

A Tabela 2 apresenta os dois modelos de emissores utilizados na pesquisa, com suas características técnicas.

Tabela 2. Características técnicas dos modelos com fluxo autocompensante utilizados no experimento.

\begin{tabular}{ccccc}
\hline Fabricante & Modelo & Vazão & $\begin{array}{c}\text { Diâmetro } \\
\text { nominal } \\
\text { mm }\end{array}$ & Pressão \\
\hline NAANDAN & $\begin{array}{c}\text { Naan PC } \\
\text { (laranja) }\end{array}$ & 3,8 & 16 & $100-350$ \\
Naan PC (azul) & 2,1 & 16 & $100-350$ \\
\hline
\end{tabular}


As mangueiras com espaçamento original entre os emissores foram cortadas e remontadas, utilizando uniões de mangueira, resultando em um espaçamento único entre gotejadores, de $0,20 \mathrm{~m}$ para todos os modelos. A pressão de serviço foi mantida em $150 \pm 15$ $\mathrm{KPa}$, por meio de um registro de pressão, colocada na linha principal de bombeamento da bancada.

O experimento foi montado em esquema fatorial $2 \times 2 \times 7$, sendo duas doses de Fe (escolhidas em função da Tabela 1), dois modelos de gotejadores e sete tempos de funcionamento com três repetições, totalizando 84 parcelas experimentais. Inicialmente foi verificada a normalidade dos dados pelo teste de Shapiro-Wilk $(\mathrm{P}<0,05)$ e a homogeneidade de variância pelo teste de Levene. Efetuou-se a análise de variância pelo teste F. As médias das vazões dos gotejadores foram comparadas pelo teste de $\mathrm{T}$ de Student a $5 \%$ de probabilidade. As interações significativas das vazões dos gotejadores com o tempo foram analisadas com base no método estatístico "Intervalo de Confiança para Diferenças entre as Médias" a $95 \%$ de probabilidade( $\left(\mathrm{IC}_{95 \%}\right)$.

Utilizou-se no experimento a água proveniente do lago do Departamento de Engenharia Rural. Foi realizada análise de água no início do experimento conforme a Tabela 3 e diariamente eram feitas análises de ferro na água para monitoramento das doses.

Tabela 3. Análise da água

\begin{tabular}{ccc}
\hline Parâmetros & Unidade & Análise \\
$\mathrm{N}$ & $\mathrm{mg} \mathrm{L}^{-1}$ & 7,0 \\
$\mathrm{P}$ & $\mathrm{mg} \mathrm{L}^{-1}$ & 4,0 \\
$\mathrm{~K}$ & $\mathrm{mg} \mathrm{L}^{-1}$ & 6,0 \\
$\mathrm{Ca}$ & $\mathrm{mg} \mathrm{L}^{-1}$ & 0,0 \\
$\mathrm{Mg}$ & $\mathrm{mg} \mathrm{L}^{-1}$ & 0,0 \\
$\mathrm{~S}$ & $\mathrm{mg} \mathrm{L}^{-1}$ & 5,0 \\
$\mathrm{Cu}$ & $\mathrm{mg} \mathrm{L}^{-1}$ & 0,0 \\
$\mathrm{Fe}$ & $\mathrm{mg} \mathrm{L}^{-1}$ & 0,51 \\
$\mathrm{Mn}$ & $\mathrm{mg} \mathrm{L}^{-1}$ & 0,0 \\
$\mathrm{Zn}$ & $\mathrm{mg} \mathrm{L}^{-1}$ & 0,0 \\
$\mathrm{pH}$ & & 6,90 \\
$\mathrm{C} . \mathrm{E}$. & $\mathrm{mS}$ & 0,022 \\
\hline
\end{tabular}

A água, após passar através dos gotejadores, era recolhida por uma calha que a reconduzia ao reservatório, constituindo, assim, um sistema de recirculação de água. No sentido de agilizar a medição da vazão dos gotejadores, era utilizada uma prateleira móvel, a qual permitia a inserção de uma bateria de 75 recipientes volumétricos graduados de $1000 \mathrm{ml}$.

O experimento foi conduzido durante 1 ano, totalizando 4800 horas de funcionamento da bancada, sendo 1200 horas para cada modelo em cada tratamento. Para cada modelo de tubo gotejador, foram efetuadas duas aplicações de dosagens de $1,5 \mathrm{mg} \mathrm{L}^{-1}$ e $3,0 \mathrm{mg} \mathrm{L}^{-1}$ de ferro, contabilizando 4 tratamentos. 
As medições individuais da vazão dos gotejadores foram efetuadas semanalmente, correspondendo a intervalos de 112 horas de funcionamento do sistema, totalizando onze medições no período de ensaio para cada tratamento. $\mathrm{O}$ volume de água coletado em 10 minutos foi determinado indiretamente através do método gravimétrico, utilizando-se uma balança de precisão Gehaka BK 4000 (0,01 g) certificada com o objetivo de se obter maior exatidão do volume coletado de cada emissor, e seus valores expressos em $\mathrm{L} \mathrm{h}^{-1}$.

Depois de tabulados os pesos, procederam-se os cálculos da vazão, do coeficiente de uniformidade de Christiansen e do coeficiente de uniformidade de distribuição através das equações 1 a 5 .

$v=\frac{M}{8}$

Em que:

$\mathrm{v}$ - volume de água, em $\mathrm{ml}$;

$\mathrm{M}$ - peso da água coletada, em g;

$\delta$ - peso específico da água utilizada no ensaio, em $\mathrm{g} \mathrm{ml}^{-1}$.

$q=\frac{(6 \times v)}{1000}$

Em que:

$\mathrm{q}$ - vazão do gotejador, em $\mathrm{L} \mathrm{h}^{-1}$;

$\mathrm{v}$ - volume de água, em $\mathrm{ml}$.

$\bar{q}=\frac{q_{\text {linh } \alpha}}{N}$

Em que:

$\mathrm{q}$ - vazão média do gotejador, em $\mathrm{L} \mathrm{h}^{-1}$;

$\mathrm{q}_{\text {linha }}$ - vazão da linha gotejadora, em $\mathrm{L} \mathrm{h}^{-1} \mathrm{e}$

$\mathrm{N}$ - número de emissores da linha.

$C U D=100 \times \frac{q_{25 \%}}{\bar{q}_{\text {wado }}}$

Em que:

CUD - coeficiente de uniformidade de distribuição, em \%;

q 25\% - vazão média de $1 / 4$ dos menores valores, em L h-1

$\mathrm{q}$ usado - vazão média do gotejador usado, em $\mathrm{L} \mathrm{h}^{-1}$.

$$
C U D=100 \times\left[1-\frac{\sum_{\bar{i}=1}^{n}|Y i-\bar{Y}|}{n \times \bar{Y}}\right]
$$

Em que: 
CUC - coeficiente de uniformidade de Christiansen, em \%;

$\mathrm{n}$ - número de amostras na linha lateral;

$\mathrm{Yi}$ - vazão do emissor, em $\mathrm{L} \mathrm{h}^{-1}$;

$\mathrm{Y}$ - vazão média dos gotejadores, em $\mathrm{L} \mathrm{h}^{-1}$.

\section{RESULTADOS E DISCUSSÃO} CUD.

No Tabela 4 apresenta os resultados da análise de variância dos valores de CUC e

Tabela 4. Análise de Variância das variáveis avaliadas: Coeficiente de Uniformidade de Distribuição (CUD) e Coeficiente de Uniformidade de Christiansen (CUC).

\begin{tabular}{|c|c|c|c|c|c|c|c|}
\hline \multirow{2}{*}{$\mathrm{FV}$} & \multirow{2}{*}{$\begin{array}{l}\text { G. } \\
\text { L. }\end{array}$} & \multicolumn{3}{|c|}{ CUD } & \multicolumn{3}{|c|}{ CUC } \\
\hline & & SQ & QM & $\mathrm{F}$ & SQ & QM & $\mathrm{F}$ \\
\hline Doses de Fe & 1 & 0,0228 & 0,0228 & $0,0392^{\mathrm{ns}}$ & 0,0430 & 0,0430 & $\underset{\substack{n s \\
\mathrm{~ns}}}{0,3068}$ \\
\hline $\begin{array}{l}\text { Tipo de } \\
\text { Gotejadores }\end{array}$ & 1 & 68,5061 & 68,5061 & $117,3186^{*}$ & 11,0659 & 11,0659 & $78,949 *$ \\
\hline Tempo & 6 & 28,6266 & 4,7711 & $8,1706^{*}$ & 6,8053 & 1,1342 & $8,0920^{*}$ \\
\hline $\begin{array}{l}\text { Doses Fe x Tipo } \\
\text { de Gotejadores }\end{array}$ & 1 & 0,0185 & 0,0185 & $0,0318^{\mathrm{ns}}$ & 0,0226 & 0,0226 & $0,1612^{\mathrm{ns}}$ \\
\hline $\begin{array}{l}\text { Doses Fe } \mathrm{x} \\
\text { Tempo }\end{array}$ & 6 & 0,6873 & 0,1145 & $0,1962^{\mathrm{ns}}$ & 0,1994 & 0,0332 & $0,2371^{\mathrm{ns}}$ \\
\hline $\begin{array}{l}\text { Tipo de } \\
\text { Gotejadores } \mathrm{x} \\
\text { Tempo }\end{array}$ & 6 & 6,1766 & 1,0294 & $1,7629^{\mathrm{ns}}$ & 1,8629 & 0,3105 & $\underset{\mathrm{ns}}{2,2151}$ \\
\hline $\begin{array}{l}\text { Doses Fe x Tipo } \\
\text { de Gotejadores } \mathrm{x} \\
\text { Tempo }\end{array}$ & 6 & 1,2816 & 0,2136 & $0,3658^{\mathrm{ns}}$ & 0,4546 & 0,0757 & $0,5405^{\mathrm{ns}}$ \\
\hline Resíduo & 56 & 32,7002 & 0,5839 & - & 7,8493 & 0,1402 & - \\
\hline Média & & & 95,3598 & & & 96,9381 & \\
\hline CV $(\%)$ & & & 0,8200 & & & 0,3220 & \\
\hline
\end{tabular}

* Significativas ao nível de 5\% de probabilidade. ns- Não significativo ao nível de $5 \%$ de probabilidade.

Nas Figuras 2 e 3, estão apresentados os valores médios de CUC para os tubogotejadores NaanPC Laranja e Azul, em função do tempo de operação do sistema de irrigação, nas doses de $1,5 \mathrm{mg} \mathrm{L}^{-1}$ e $3,0 \mathrm{mg} \mathrm{L}^{-1}$, respectivamente. 


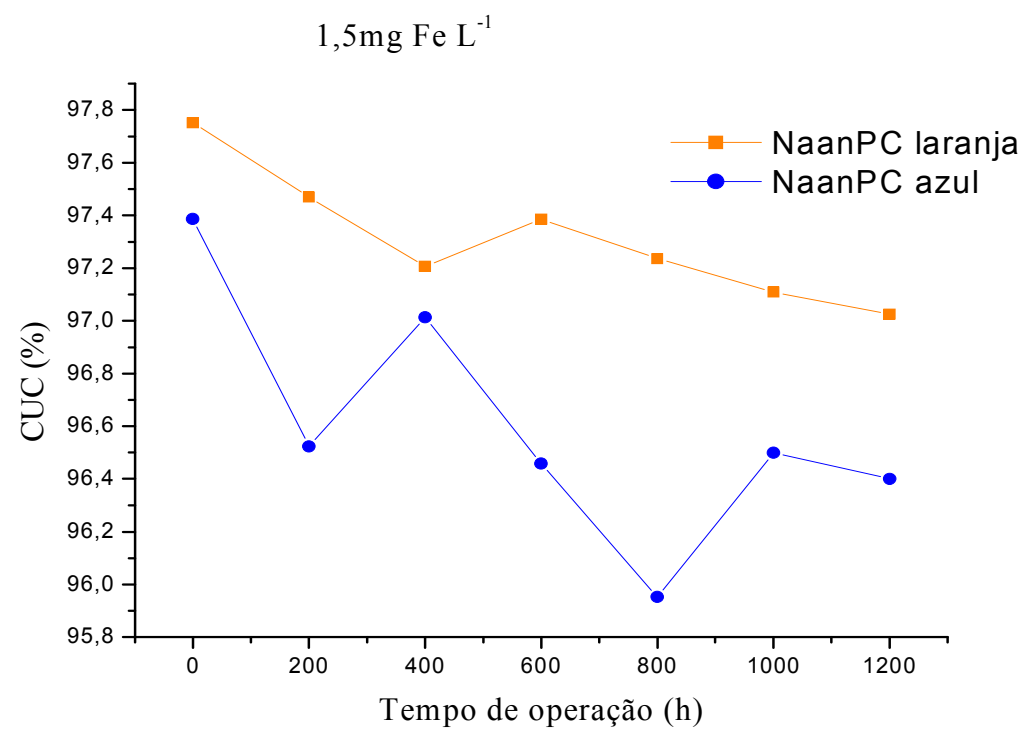

Figura 2. Valores médios de CUC, para os tubo gotejadores NaanPC Laranja e NaanPC Azul, em função do tempo de operação, na dose de $1,5 \mathrm{mg} \mathrm{L}^{-1}$ de ferro total.

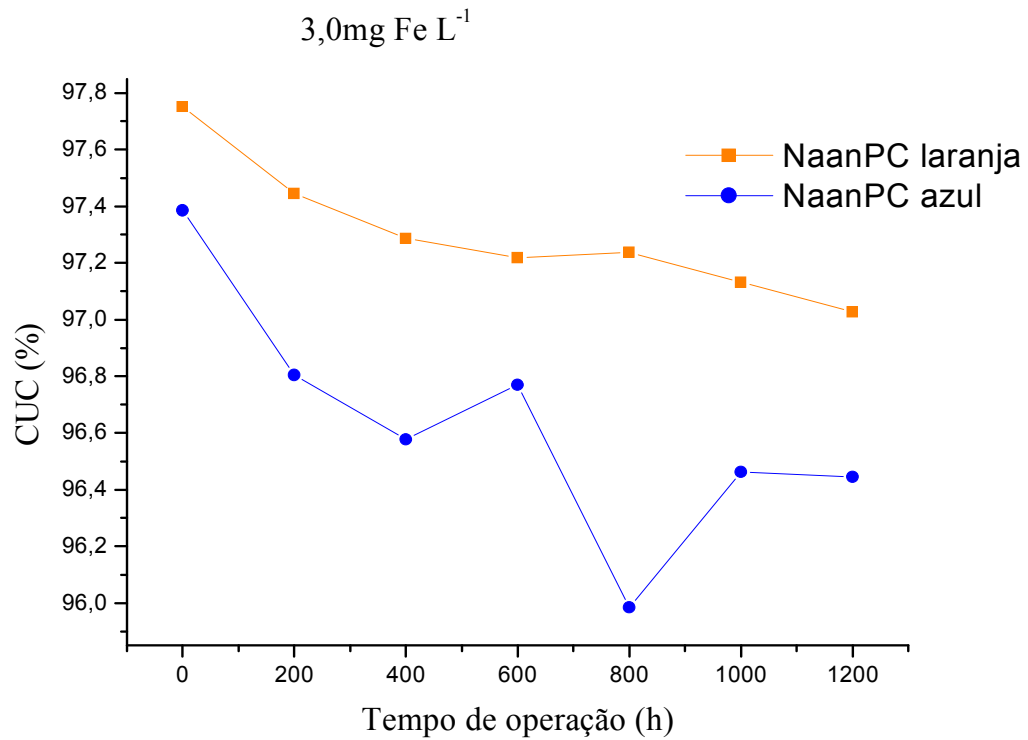

Figura 3. Valores médios de CUC, para os tubo gotejadores NaanPC Laranja e NaanPC Azul, em função do tempo de operação, na dose de $3,0 \mathrm{mg} \mathrm{L}^{-1}$ de ferro total.

Observa-se na Figura 2 uma redução nos valores médios do CUC, para os dois gotejadores, ao longo do tempo de operação do sistema de irrigação, com a utilização de água ferruginosa. Os valores médios do CUC para os modelos NaanPC laranja e NaanPC azul apresentaram reduções de $0,74 \%$ e $1,48 \%$, respectivamente, o que permite mantê-los na classificação de excelente, mesmo após 1200 horas de operação do sistema de irrigação.

Observa-se na Figura 3 também, uma redução nos valores médios do CUC, para os dois gotejadores, ao longo do tempo de operação do sistema de irrigação, com a utilização de 
água ferruginosa. Os valores médios do CUC para os modelos NaanPC laranja e NaanPC azul apresentaram reduções de $0,74 \%$ e $1,44 \%$, respectivamente, o que o também permite mantêlos na classificação de excelente, mesmo após 1200 horas de operação do sistema de irrigação. Tal sensibilidade ao entupimento foi atribuída à característica deste gotejador de apresentar duas saídas de água, sendo que uma delas ficava virada para cima, o que permitiu um maior acúmulo de mucilagem e/ou precipitados na câmara de autocompensação.

Estão apresentados nas Figuras 4 e 5, os valores médios do CUD para os tubo gotejadores NaanPC Laranja e Azul, em função do tempo de operação do sistema de irrigação, nas doses de $1,5 \mathrm{mg} \mathrm{L}^{-1}$ e $3,0 \mathrm{mg} \mathrm{L}^{-1}$, respectivamente.

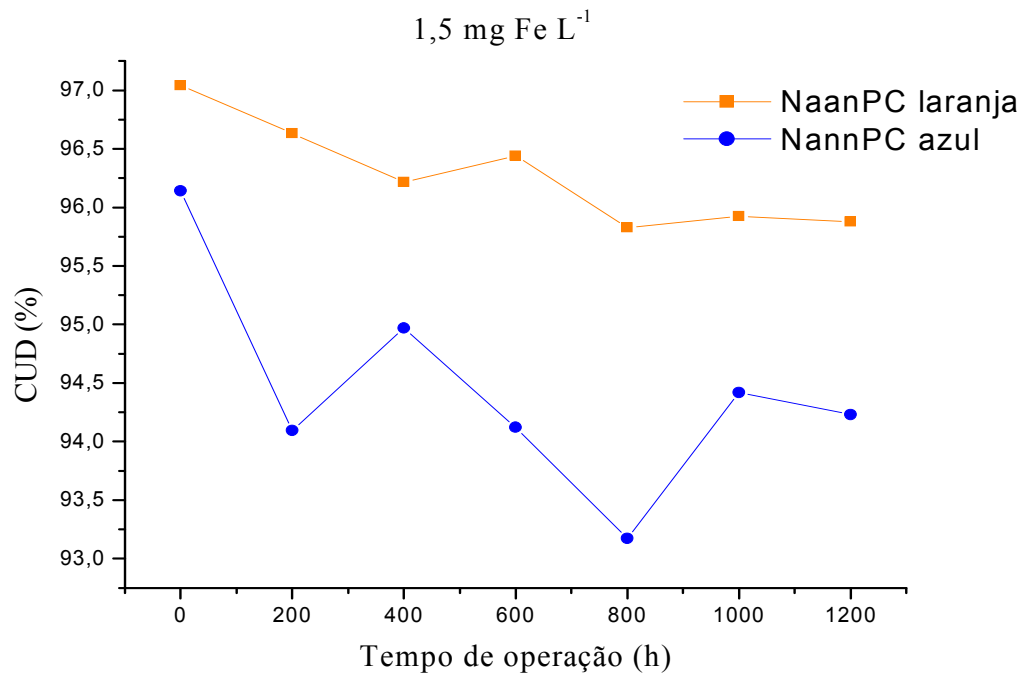

Figura 4. Valores médios de CUD, para os tubogotejadores NaanPC Laranja e NaanPC Azul, em função do tempo de operação, na dose de $1,5 \mathrm{mg} \mathrm{L}^{-1}$ de ferro total.

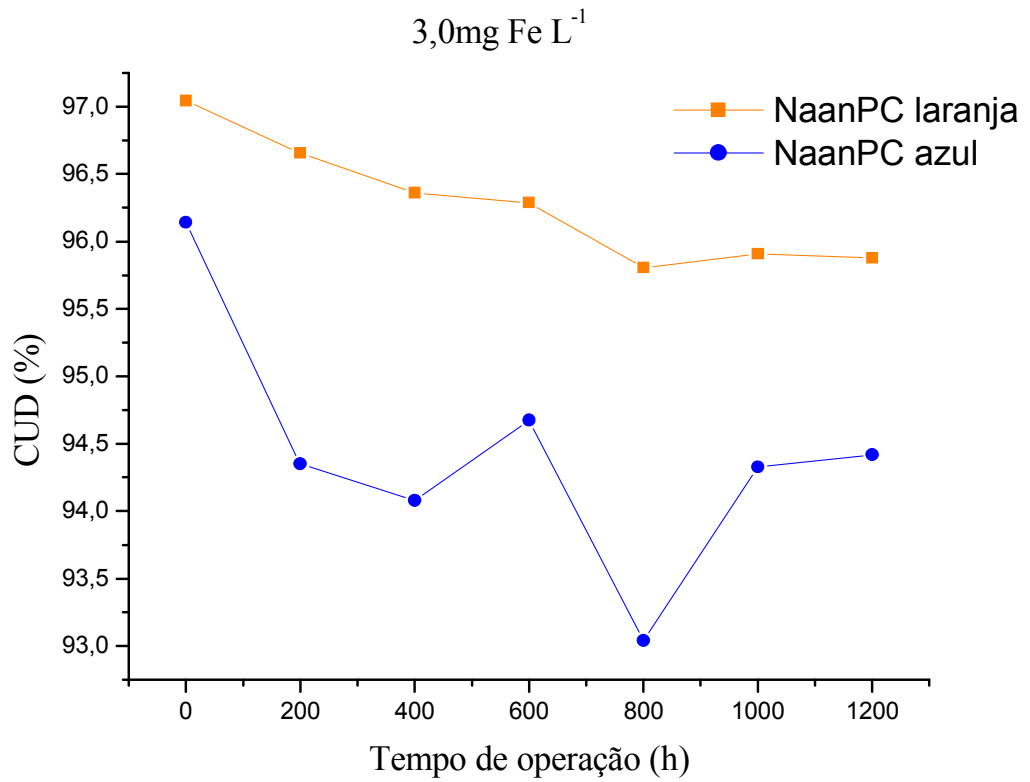

Figura 5. Valores médios de CUD, para os tubogotejadores NaanPC Laranja e NaanPC Azul, em função do tempo de operação, na dose de $3,0 \mathrm{mg} \mathrm{L}^{-1}$ de ferro total. 
O coeficiente de uniformidade de distribuição (CUD) mostrou-se mais sensível as variações de uniformidade de aplicação de água pelo efeito do entupimento que o coeficiente de uniformidade de Christiansen (CUC), como era de se esperar, pois em seu cálculo, utilizam-se valores médios dos $25 \%$ dos menores valores das vazões.

$\mathrm{Na}$ primeira avaliação, os valores médios do CUD permitiram a classificação excelente para os dois modelos de gotejadores nas duas doses de ferro total. Observa-se na Figura 4 uma redução nos valores médios do CUD, para os dois gotejadores, ao longo do tempo de operação do sistema de irrigação, com a utilização de água ferruginosa. Os valores médios do CUD para os modelos NaanPC laranja e NaanPC azul apresentaram reduções de $1,25 \%$ e $3,09 \%$, respectivamente, o que permite mantê-los na classificação de excelente, mesmo após 1200 horas de funcionamento do sistema de irrigação.

Observa-se também na Figura 5, uma redução nos valores médios do CUC, para os dois gotejadores, ao longo do tempo de operação do sistema de irrigação, com a utilização de água ferruginosa. Os valores médios do CUD para os modelos NaanPC laranja e NaanPC azul apresentaram reduções de $1,28 \%$ e $2,15 \%$, respectivamente, o que o também permite mantêlos na classificação de excelente, mesmo após 1200 horas de funcionamento do sistema de irrigação.

\section{CONCLUSÕES}

Os resultados obtidos na condução do ensaio permitiram chegar às seguintes conclusões:

a) Com o tempo de utilização de água ferruginosa nas diferentes doses, os valores médios do Coeficiente de Uniformidade de Christiansen (CUC) diminuíram para os dois modelos de tubogotejadores, porém ambos ainda mantiveram a classificação de excelente, mesmo após 1200 horas de funcionamento do sistema de irrigação; e

b) O coeficiente de uniformidade de distribuição (CUD) mostrou-se mais sensível ao entupimento, pois em seu cálculo utiliza a média dos $25 \%$ menores valores das vazões. Entretanto mesmo sendo mais sensível, em todas as avaliações manteve a classificação de excelente, mesmo após 1200 horas de funcionamento do sistema de irrigação.

\section{REFERÊNCIAS}

BERNARDO, S. Manual de irrigação. Viçosa: Imprensa Universitária, 1995. 657p.

BUCKS, D. A.; NAKAYAMA, F. S.; GILBERT, R.G. Trickle irrigation water quality and preventive maintenance. Agricultural Water Management, v.2, p.149-162, 1979.

MANTOVANI, E.C.; RAMOS, M.M. Manejo da irrigação. In: COSTA, E.F.; VIEIRA, R.F.; VIANA, P.A. Quimigação: aplicação de produtos químicos e biológicos via irrigação. Brasília, DF; EMBRAPA, Centro de Pesquisa de Milho e Sorgo, 1994. p. 129-158.

POVOA, A. F.; HILLS, D. J. Sensitivity of microirrigation system pressure to emitter plugging and lateral line perfurations. Transactions of the ASAE, St, Joseph, v. 37, n.3, p. 793-799,1994. 
REIS, E.F.; et al.. Uniformidade de aplicação de água em sistema de irrigação localizada no sul do Estado do Espírito Santo - ES. In: CONGRESSO BRASILEIRO DE ENGENHARIA AGRÍCOLA, 31, 2002, Salvador. Anais... Salvador: UFBA/SBEA,2002. 1CD - ROM. 